\title{
Survey of Optimized CSP Migration
}

\author{
Kadlag R A ${ }^{1}$, Devikar R N ${ }^{2}$ \\ ${ }^{1}$ Department of Information Technology, Amrutvahini College of Engineering, Sangamner, (M.S) India-431005 \\ ${ }^{2}$ Department of Information Technology, Amrutvahini College of Engineering, Sangamner, (M.S) India-431005
}

\begin{abstract}
Cloud computing allow the different business to emerge upon a large scale, it provide a large computing environment, space, application along with the storage space to the business. Thus allow scaling up the business and slowing down their resource to maintain and stored. More over the cloud environment works on the base of the gain in virtualization of the resources over a network so generally it is compared by grid computing. But it also raises the scale of the server to concern, to manage and to use the application to exabyte, when the service is to be migrated on to the different CSP. The system allows the user to select different CSP or datacenters to manage and store the data but, when migration is done the system needs to retrieve the whole data stored onto their system, which also can allow more faults and process failures to the system. This may lead the system to crash or go in data-lock in condition. To avoid this problem here a survey is done of the system's that are designed to allows user to select their CSP according to their needs on demand, as well as it allow user to select the CSP to migrate on. The transfer of data in the system allows user to transfer whole data to CSP that they had demanded with the provision of fault-tolerance and reliability of data transferred on the new CSP. Even blanks can be filled, as in this survey we also provide TPA relations that acts a middle tier to the user and the CSP that rates the CSP accordingly to the logs. Thus the survey enlightens the performance and experiments of the system.
\end{abstract}

Keywords: Cloud Computing, CSP, fault- tolerance, grid computing, migration, optimization, reliability

\section{Introduction}

\section{A. Cloud Computing}

Cloud Computing allows user to use demanded services to their system. It allows user to use software as service, Infrastructure as a service, Platform as a service. That is it gives complete cornucopias of computational resources and environment [1], [2]. Using IAAS and PAAS, the cloud has given a large amount of relief to the money and time of the large emerging Web developers like dropbox, icloud, etc [3]. In short, cloud computing provides virtualization, storage, resources in network as per demand. Not only this cloud computing also allows to convey the necessary services for economical and some of technological needs. Thus cloud computing yield market on rapid base in business markets.

Cloud also has inbuilt services which are technically vital in networking such as: Efficient, reliable, optimization, resource availability, management, portability etc [3], [4]. Due to these reasons cloud computing gets to continue to grow on large scale in terms of infrastructure, software as well as hardware [5]. Also it has gain large attention of numbers of researcher to work and survey on in its field.

\section{B. CSP (Cloud Service Providers)}

Cloud gained market in the business world just because of its service delivering task may the service be related to software, hardware, or resources for the platforms. It gave the entrepreneur, a wide range to expand in the both commercial and in source platforms to withstand in the updating markets.

Cloud services are on-demand services; also it gives scalability and availability, dynamic concurrency in the system. It also assures the security, virtualization, recovery of the data stored in system [4]. Although cloud provides the service and management along with this problem of reliability and data integrity comes in hand. It also imposes lot of cost and time consuming factors to the client system
[5]. Also the relaxation of storage space and installation charges (especially for the licensed versions software) is reduced in cloud.

It provide user to dynamically connect to internet and perform their task instantly to get result dynamically to all the co users of the system [6]. But it doesn't allow the user the satisfaction of reliability and integrity of the data. Thus, even when organizations that deploy cloud-based applications could be surprised if they decide to change cloud service providers (CSP). The lack of data interchange standards and the sluggish speed of bulk data transfers over TCP/IP networks make data portability between clouds difficult at best - and unworkable at worst of it. Now a day many emerging companies are entering in providing best CSP (EMC, AMAZON etc), that give a trust worthy environment in different cloud (Hybrid, Private, Public).

Larry $\mathrm{F}$ [9] research on this and states that data portability is one of the slug thing in the cloud that can be resolved, the unsatisfaction to the data and service may led to the bankruptcy and loss in business. But these dynamical allocations of data centers and resources in it make the system more complex to manage and maintain. This area is in need to survey more in resolution of operational complexity and scalability, to increase the performance of the cloud systems. Also management of increasing traffic around the cloud needs to be focus on.

So, as to reboost the system throughput. The survey here focuses on CSP to manage these services and complexity in the cloud environment.[10] Cloud Service Provider (CSP) manages the operations in between cloud and user. The user gives their data to CSP; CSP has control on the data.

Also every time the user needs to make sure the data appears is correct and vigilant to the original, on the cloud. A threat for data integrity is again a problem generally faced in cloud

\section{Volume 4 Issue 12, December 2015}




\section{International Journal of Science and Research (IJSR) \\ ISSN (Online): 2319-7064}

Index Copernicus Value (2013): 6.14 | Impact Factor (2014): 5.611

for CSP might behave unfaithfully. For money reasons, CSP might can change the priority of data according to the accessed period also it might delete the data that are sparsely used for availability of storage and working space.

\section{Optimization in Cloud}

Generally, the optimization in cloud allows several SLA's to impose on the cloud. It makes the services in the cloud to scale, on high availability and performance related to the cloud data. [11] The design and operation of any system needs optimization or process to make it as good as possible in some defined sense.

To gain more fast and accurate services CSP need to be deployed in such a way that can allow dynamic way in routing optimization technology, which identifies fast, reliable use of cloud data routing in real time as user requests come in. [12 - 16] Optimization can be obtain in cloud environment under the different environment by using WAN and LAN services in networks of server for increasing the CPU time and processing speed, also by increasing the network applications impacts and prioritization of different application on network (according to the poor or fast performance of application), Optimization can be also increased by minimization of cost for the cloud deployment issues and post related to the deployment.

Cloud monitoring according to performance, scalability of data, reliability, network bandwidths can also put and helping hand in the deployment of optimized cloud environment. Also, the storage in cloud can be optimized by increasing data storing and accessing performances.

This paper survey on the above related issues. So generally we can say that optimization of cloud computing environment can be rated as: 1) to align between the two operations, the software and business to related to it. 2) Improvements in the operational and the management's tools required. 3) Making of the business with consoled cost accordingly. 4) Best checking practice for better service and business vulnerabilities.

\section{Service Migration}

The process of transition to a different cloud service provider is known as cloud service migration. Cloud service can be according to the demand of client but also impose the charges to that demand which can differ from cloud service to service.

When a user migrate from one cloud service to the other provider, the data in use of the client can impose the extra charge by the CSP to mitigate from one cloud to another, this charges can directly be discarder using the technique called service migration of a client instead of data migration.

[17] A study that considers check of the cloud data for migration before it is migrated as a best solution to the service for data migration in cloud. The data need to be migrated is send to a virtual cloud from physical their data and software is checked for the integrity and smooth running of the process, this gives the assured result for data integrity while migration of the data over cloud. The traditional migration system includes complete transfer of data to the users system and then the migration or uploading of data to the desired system, at once [18]. This requires lot of runtime delays for the other application in work. When it comes to the Web application the companies can face a long loss because of server delay.

Thus before migration of any data and services in the cloud we need to make sure about the performance and operational fairness in on the system. Also, we need to check the current system and the selected system as up to benchmark position. Also the necessity of survey and research is needed over the points of migration of fully secured and visible data stack provided by user. The performance measures of migration system to document success in that system.

\section{Related Work}
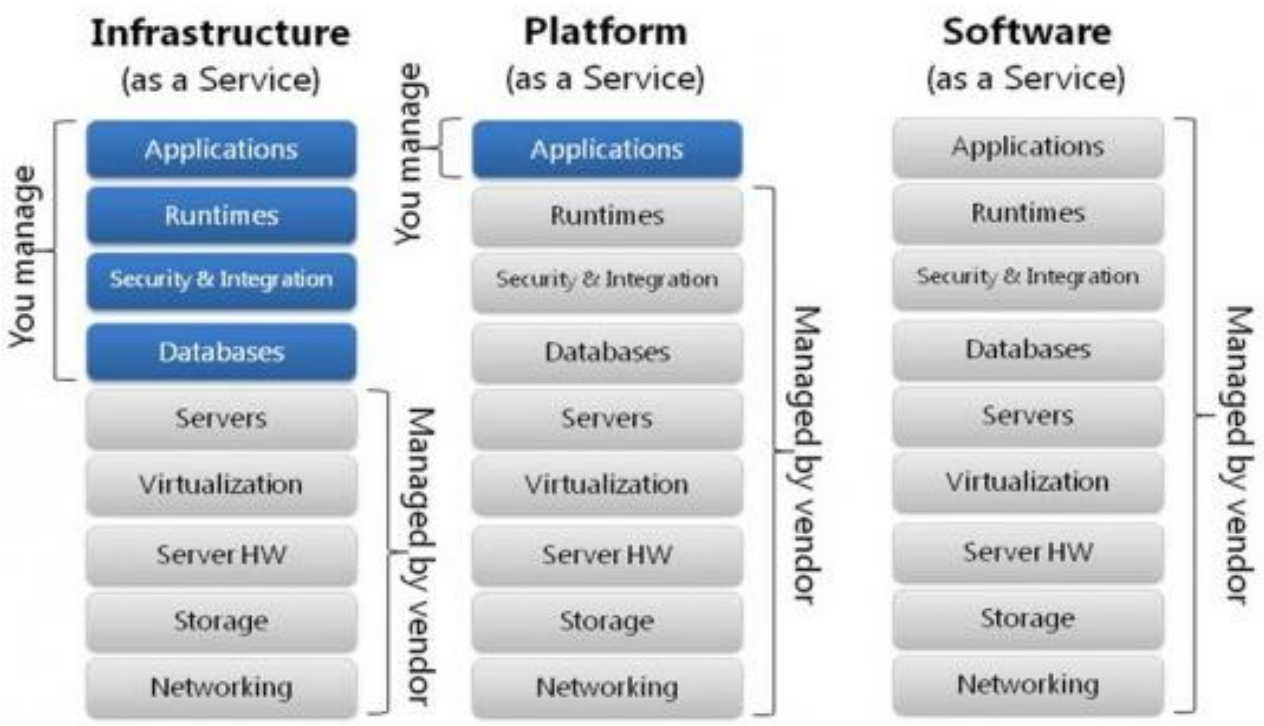

Figure 1: Cloud Computing 


\section{International Journal of Science and Research (IJSR) \\ ISSN (Online): 2319-7064}

Index Copernicus Value (2013): 6.14 | Impact Factor (2014): 5.611

Cloud computing has hosted many business attentions due to the provision of its as a Service" tags, that to on a large scale with the easy to use facility. Cloud computing is sometimes viewed as a reincarnation of the classic mainframe client-server model.

However, resources are ubiquitous, scalable, highly virtualized. The system designed yet till now focus mainly only on gaining of more and more services from cloud in their platforms, but the issues and challenges faced in later era gave a boost to surveys and research in the evaluation and remedy measures to sustain in, within the cloud.

The applications, runtimes, database and processing related to data are generally controlled by the cloud systems itself hence is considered as the software data reliving technology. The cloud acts as a black hole where nothing inside the cloud is visible to the clients. Clients have no idea [8] or control over happening in that cloud.

Even if the CSP is trustful, it can have malicious system and administration that can tamper of the content of the client violating the data integrity and confidentiality in between the cloud. Clouds is still under the fear of dogmatism for traditional data confidentiality, integrity, availability, and privacy issues, plus some additional attacks. Recently many works are in continuations that has served a milestone in the way to secure data integrity and storage security in cloud [8], [9], [20- 26].

Here, a survey related to better performance of Data integrity checking, Data reliability, Migration of data and migration of services in cloud are stated.

[23] A PDP module is suggested to improve the data integrity checking in the system for trust worthy CSP. A concept of homomorphic tags in the building blocks of data are considered while storing the data to the cloud. These tags are then after checked by the CSP to check the integrity of data while downloading the data to the server for use. But, this system fails in data privacy preservation and is not dynamic.

[24] Here, the paper suggests the POR and a singleton set of key for cryptography. Also CSP needs to access few portion of data sets at time of archiving of file. Thus the protocols suggests to encrypt the data block with a certain key and check the validation while retrieving of that file. But the system limits the query actions. Also prevent the dynamic checking of the data.

Q wang. [25] suggested the schemes by allowing the TPA to act as a mediator for the system to work with the Service providers (CSP), on behalf of user to communicate and get dynamic data storage and integrity facility of the cloud. Here a brief counting on method on MHT is done to modify the storage of data and to improve the data integrity. But the scheme does not provide full privacy preservation.

So, in year $2011 \mathrm{Z}$. hao [26] suggest a ideal system that uses the protocol based on homomorphic verification tags that are deployed to set up with few algorithms such as SigGen, GenProof, TagGen, Checkproof that can stand proof for the challenges faced in data integrity.

He suggested to allows pair of keys that describe for Dm no of data blocks that are stored in the cloud. Accordingly as the priority for the backup can be facilitate in the cloud. This system also had proved under the features related to security, TPA verification, and dynamic block storage of data.

Table 1: Comparative study for the data integrity protocols

\begin{tabular}{|c|c|c|c|c|}
\hline & {$[23]$} & {$[24]$} & {$[25]$} & {$[26]$} \\
\hline Privacy & $\chi$ & $\nearrow$ & $\chi$ & $\checkmark$ \\
\hline Security & $\checkmark$ & $\checkmark$ & $\checkmark$ & $\checkmark$ \\
\hline Data dynamics & $\chi$ & $\chi$ & $\checkmark$ & $\checkmark$ \\
\hline $\begin{array}{c}\text { Trusted } \\
\text { TPA }\end{array}$ & $\chi$ & $\chi$ & $\checkmark$ & $\checkmark$ \\
\hline
\end{tabular}

Reliability of data is the Qos that is provided with the data during the system is deployed. Lee gave the idea for ensuring the Qos by a software approach. As per the study, reliability of the data do not come for free it delivers charge on scalability of the cloud. [5] The simple way is to just upload the data to the cloud and add it to the resource available. But there are other schemes that can assure the high Qos with cost-effective environment.

Replication of data is one of the easy solutions applied for the reliability. It helps in backup of file that can prove against the vulnerable changes that can be made in the documents after a lease of time. it can also give secure additional database and reduce the Qos lowering[6]. Generally logs are used to monitor the reliability of the system. In the traditional schemes the reliability thus needed the logs of the system to monitor on continuously. While deploying this, server need to update the logs and monitor it, hence it needed large space for storage of the logs.

[7] The scheme that handles this scheme imposing different SLA's for the verification are used. The SLA guarantees the client verification while storing and retrieving the data. Smom SLA also assures that the client can see the data that is verified by the system and he is allowed to view.

[8] Here, the reliability of the scheme is to use the Fault tolerance mechanism where, the design of the system is according with hand to the replication of the stored data. Here the components to e replicated are selected according to the priority by the user. Depending upon the vitalness of the stored data. Thus when the data is been stored it checks for the updating of the priority of the data, also the system ensure that the client is verified for the access of the data.

Thus the failure impact of the system is first checked to make the system fault tolerant and reliable:

$$
\begin{array}{ll}
\mathrm{FT}\left(\mathrm{n}_{\mathrm{i}}\right)=\frac{\mu\left(\mathrm{n}_{\mathrm{i}}\right)}{\sum \mathrm{N}_{\mathrm{ij}}} & \text { Equation (1) }
\end{array}
$$

Where, $\mathrm{FT}\left(\mathrm{n}_{\mathrm{i}}\right)$ is the failure of nodes in the system, $\mu\left(\mathrm{n}_{\mathrm{i}}\right)_{-}$is the sum of the number of nodes failed, $\mathrm{N}_{\mathrm{ij}}$ is the nodes that are edged from $n_{i}$ to $n_{j}$.

\section{Volume 4 Issue 12, December 2015}




\section{International Journal of Science and Research (IJSR) \\ ISSN (Online): 2319-7064}

Index Copernicus Value (2013): 6.14 $\mid$ Impact Factor (2014): 5.611

[19] The general motivation for cloud migration is to gain more of Broader reach in terms of number of clients, to ease the mobility access of the data around the world, Business agility and flexibility, to give broad range of business market also, to improved level of security than the previous system, that can deliver the improved response time of the system. Also the provision of reduction of relocation data cost is under consideration.

[20] The paradigm that are suggested here allows user to replicate the service around number of nodes, thus optimizing the load around number of nodes.

The system here can go in the cycle lock if the system edge the service that is virtual and is a false node created by the different providers to gain the benefits of acquiring the secured data to their system.

Here, the system needs to go through the response time log to monitor the nodes around for better performance. Thus the system is not secured in terms of data confidentiality. Also the system automatically resumes the migration from the neighboring node after completion of the task.

Also Cloudsim provide the migration of service with simulation of available node around the system nearby, the ACO system algorithm applies best to the task but, doesn't give the assured for the reliable data nodes to be in the network while in use. Cloud simulator also impose more charges to the client while it provide the data center to the client.

[21]The migration of the data is classified here in two types: big bang migration, trickle migration. Both the techniques involve the wait-time while migrating the data to the other selected cloud environment. Big bang Algo. Stop the complete system under work and download the data to the system of client. While trickle migration Algo. Provide the facility to stop the only working component where migration of the data is proceeding.

[22] The service migration here discussed allows user to relocate the complete data and the service provided by the CSP to the other cloud. Thus the other node where the system is placed can provide user with better Qos. Due to this the performance rates of the system increase. Also the provision is made to see that the imposing charge for the migration is minimized according to the need of the client. The node already in use then becomes unsustainable to the particular client, keeping the integrity of the system. Thus the service migrated to the maximum response time server.

\section{Proposed System}

Proposed system allows enabled the auto-scaling feature. Based on this, implementation a benchmark to measure the performance of our Cloud service provider is been created. Also the provision of service migration and data transfer from one cloud to other can be done.

The system to be developed will also help users in finding optimized solution for cloud migration as per the cost and time management to provide client service provider among the different providers.

The system is intended to provide migration mechanism which can be easily implemented in the cloud computing environment for the efficient, robust and cost effective management. On giving a specification related to optimization factors system will offer list of better options to user along with the analysis report.

It also increases agility not only by standardizing the provisioning process, but also by addressing the computing resources life cycle. The Proposed system can be achieved using the packaging of complete data in a pack and then transferring it to the other cloud node.

This system includes the transfer of service from one cloud server to another accordingly to user demand. It allows the complete transfer of data from one server to another as soon as the service is changed. A typical, SLA imposes specific levels of the services such as availability of the services which are in operation. SLA includes the client authentication for a particular cloud server. As well as data uploading according to storage space of server. Although cloud providers bring advantages to their users, most cloud providers user outages from time-to-time. Therefore, relying on a single cloud storage services threatens service availability of cloud users. By proposing a system based an algorithm that determines the minimum replication cost of objects such that the expected availability for users is guaranteed. 


\section{International Journal of Science and Research (IJSR) \\ ISSN (Online): 2319-7064}

Index Copernicus Value (2013): 6.14 | Impact Factor (2014): 5.611

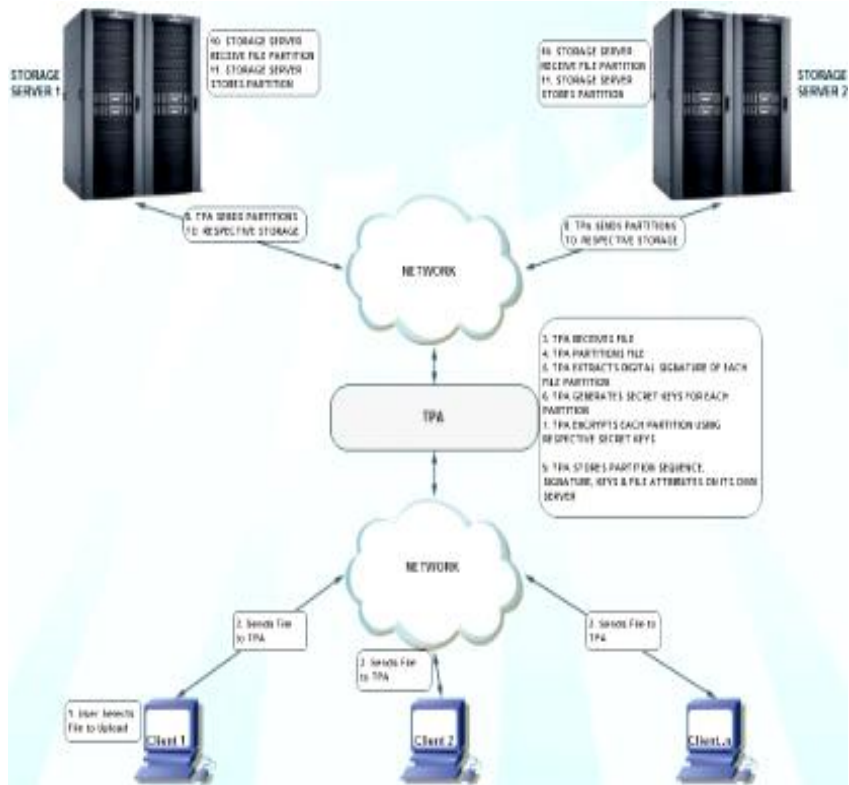

Figure 2: System architecture of the proposed system

\section{Conclusion}

In this paper, survey related to the cloud migration and data reliability issues are discussed. We compared the existing system to the newly proposed system for better performance. Here the system can guarantee that TPA would not learn any knowledge about the Data content stored on the cloud server during the efficient Auditing process, which not only eliminates the burden of Cloud user from the tedious and possibly expensive auditing Task, but also alleviates the users' fear of their outsourced Data leakage.

\section{References}

[1] P. Jain, Security issues and their solutions in cloud computing." International journal for computing and business research, ISSN: 229- 6166.

[2] Rabi. Padhy, Manas. Patra, Suresh. Statapathy, Eloud Computing: Security issues and challenges", IJCSITS, vol. 1, no. 2, Dec. 2011.

[3] Jeff. L., - Cloud Computing for Optimization", http://www.mcs.anl.gov/MACS/files/2013/01/linderothICS2013.pdf.

[4] Amrutha K. K, Madhu B. R, An Efficient Approach to find best CSP through brokers", International journal for Advanced research in Computer Science and Software Engineering, vol.4, no. 7, July 2014.

[5] Ki-Woong Park , Jaesun Han and Kyu Ho Park, THEMIS: A Mutually Verifiable Billing System for the Cloud Computing Environment," IEEE TRANSACTION ON SERVICES COMPUTING, VOL. 6, NO.3, JULY-SEPTEMBER 2013.

[6] S. Venkataramana, Estimating Trust Value for Cloud Service Providers using Fuzzy Logic", International journal for computer application, vol.7, no.19, June 2012.

[7] Z. Zheng and M.R. Lyu, Component Ranking for Fault-Tolerant Cloud Applications, "IEEE Trans. Serv. Comput. (TSC), vol. 5,no. 4, pp. 540-550, 2012.
[8] Kalyani Madhurwar, Alovel Method for Trusted Third Party Security Services for Cloud",vol. 5, no. 6, July 2014.

[9] Ebtesam A. Alomari, Muhammad M. Monowar, A Survey of Security Issues for Data Sharing over Untrusted Cloud Providers", Journal of Emerging Trends in Computing and Information Sciences, vol. 5, no. 8 , Aug. 2014.

[10] J lee -Software approach to assuring the high scalability in cloud computing", in IEEE, 2010.

[11] Z.Kerravala,http://searchenterprisewan.techtarget.com/ti p/WAN-optimization-technology-improves-cloudcomputing-performance, Techtarget network.

[12] A.jangra, Scheduling optimization in cloud computing", ijarcsse, vol. 3, no. 4. April 2013

[13] Frost and Sullivan, Essentials for csps to succeed with cloud services", https://www.ibm.com, oct-2010.pdf.

[14] Li Zhou; Yi-Cheng Wang; Ji-Lin Zhang; Jian Wan; Yong-Jian Ren, "Optimize Block-Level Cloud Storage System with Load-Balance Strategy," in Parallel and Distributed Processing Symposium Workshops \& PhD Forum (IPDPSW), 2012 IEEE 26th International, vol.4, no.3, pp.2162-2167, 21-25 May 2012.

[15] Ramamoorthy, S.; Rajalakshmi, S., "Optimized data analysis in cloud using BigData analytics techniques," in Computing, Communications and Networking Technologies (ICCCNT),2013 Fourth International Conference on, vol.4, no.6, pp.1-5, 4-6 July 2013

[16] Qinlu He; Zhanhuai Li; Bo Wang; Huifeng Wang; Jian Sun, "Research on Cloud Storage Environment File System Performance Optimization," in Information Management, Innovation Management and Industrial Engineering (ICIII), 2011 International Conference on, vol.2, no3., pp.78-83, 26-27 Nov. 2011.

[17] Goyal, V.; Kant, C., "An effective algorithmic approach for cost optimization in Cloud based data center," in Issues and Challenges in Intelligent Computing Techniques (ICICT), 2014 International Conference on, vol.4, no.5, pp.630-637, 7-8 Feb. 2014.

[18] Periasamy, R., "Performance Optimization in Cloud Computing Environment," in Cloud Computing in Emerging Markets (CCEM), 2012 IEEE International Conference on , vol.3, no.4, pp.1-6, 11-12 Oct. 2012.

[19] Oikonomou, K.; Stavrakakis, I., Scalable service migration in autonomic network environments", IEEE, Jan. 2010

[20] Goudarzi, H.; Ghasemazar, M.; Pedram, M., "SLAbased Optimization of Power and Migration Cost in Cloud Computing," in Cluster, Cloud and Grid Computing (CCGrid), 2012 12th IEEE/ACM International Symposium on , vol., no., pp.172-179, 1316 May 2012.

[21] Fareed Jokhio, Adnan Ashraf, S'ebastien Lafond, Ivan Porres, Johan Lilius Đynamic Resource allocation technique in cloud Environment"

[22] S. Al-kiswany, D. Subhraveti, P. Sarkar, and M. Ripeanu, _VMFlock: Virtual Machine Co-Migration for the Cloud," in Proc. 20th Int. Symp. High Perform. Distrib. Comput., New York, NY, USA 2011, pp. 159-170.

[23] Qian Wang, Student Member, IEEE, Cong Wang, Student Member,IEEE, Kui Ren, Member, IEEE

\section{Volume 4 Issue 12, December 2015}


Wenjing Lou, Senior Member, IEEE, and Jin Li Enabling Public Auditability and Data Dynamics for Storage Security in Cloud Computing" IEEE transactions on parallel and distributed systems, vol. 22, no.5, May 2011.

[24] P. Mell, T. Grance (2009),-Đraft NIST working definition of cloud computing", [Online]Available: http:/www.csrc.nist. gov/groups/SNS/cloudcomputing/index.html.

[25] A. Juels and B.S. Kaliski Jr., Pors: Proofs of Retrievability forLarge Files,'Proc. 14th ACM Conf. Computer and Comm. Security(CCS '07),pp. 584-597, 2007.

[26] H. Shacham and B. Waters, Compact Proofs of Retrievability" Proc. 14th Int'l Conf. Theory and Application of Cryptology and Information Security: Advances in Cryptology (ASIACRYPT '08),pp. 90107,2008 . 